\title{
Effects of Diesel Exhaust Particles on Left Ventricular Function in Isoproterenol-Induced Myocardial Injury and Healthy Rats
}

\author{
Yuan-Horng Yan \\ Institute of Occupational Medicine and Industrial Hygiene, College of Public Health, National Taiwan \\ University, Taipei, and Department of Internal Medicine, Chia-Yi Christian Hospital, Chiayi, Taiwan
}

\section{Chien-Hua Huang and Wen-Jone Chen}

Department of Emergency Medicine, National Taiwan University Medical College and Hospital, Taipei, Taiwan

\section{Ming-Fang Wu}

Labratory Animal Center, National Taiwan University Medical College, Taipei, Taiwan

\author{
Tsun-Jen Cheng \\ Institute of Occupational Medicine and Industrial Hygiene, College of Public Health, National Taiwan \\ University, Taipei, Taiwan
}

\begin{abstract}
The associations between ambient particulate matter with an aerodiameter less than $2.5 \mu \mathrm{m}$ $\left(\mathbf{P M}_{2.5}\right)$ and congestive heart failure (CHF) have been reported. However, the underlying mechanisms remain unclear. We investigated the effect of diesel exhaust particles (DEPs) on left ventricular function in isoproterenol (ISO)-induced myocardial injury and healthy rats. Male Sprague-Dawley (SD) rats were injected with ISO or normal saline. Seven days later, both groups were further assigned to receive either DEPs or normal saline by intratracheal instillation (IT). Echocardiography was used to measure fractional shortening (FS) and left ventricular end-diastolic diameter (LVDd) $24 \mathrm{~h}$ before and after IT in each rat. Fractional shortening (FS) was significantly decreased in SD rats treated with ISO as compared to those treated with normal saline ( $p<.05, t$-test). When FS and LVDd before and after treatment were compared in each rat, there was no difference for normal saline treatment in healthy or ISO groups. However, there was significantly lower FS before and after DEPs exposure in both groups $(p<.05$, paired $t$-test). When using SD rats treated with normal saline as a reference group, both SD rats treated with DEPs and ISO rats treated with normal saline had lower FS $(p<.05$ and .0001 , respectively, $t$-test), while ISO rats treated with DEPs had the lowest FS $(p<.0001$, $t$-test). Echocardiographic assessment revealed that left ventricular function was impaired by acute DEPs exposure, and this $\mathrm{LV}$ function was further compromised in rats with preexisting ISO-induced myocardial injury.
\end{abstract}

Epidemiological studies have found associations between ambient particulate matter (PM) and increased cardiovascular

Received 9 March 2007; accepted 24 April 2007.

This study was in part funded by the EPA of Taiwan (NSC 94EPA-Z-002-007). The authors thank Wei-Ju Ho for technical assistance. We are also grateful to Yu-Fang Huang and Jocelin Yo-Jud Cheng for editorial assistance.

Address correspondence to Dr. Tsun-Jen Cheng, Institute of Occupational Medicine and Industrial Hygiene, College of Public Health, National Taiwan University, 17 Xu-Chou Rd. Rm 720. Taipei, Taiwan, 10055.E-mail: tcheng@ntu.edu.tw morbidity and mortality in susceptible population, including ischemic heart diseases and arrhythmia (Brook et al., 2004; Peters, 2005). Recent studies also suggest an association between PM and the daily hospital admissions of congestive heart failure (CHF). This association appears more consistent for the PM with an aerodiameter less than $2.5 \mu \mathrm{m}\left(\mathrm{PM}_{2.5}\right)$ (Metzger et al., 2004; Dominici et al., 2006; Symons et al., 2006), as compared to the PM with an aerodiameter less than $10 \mu \mathrm{m}\left(\mathrm{PM}_{10}\right)($ Schwartz et al., 1995; Morris et al., 1998; Koken et al., 2003; Wellenius et al., 2005, 2006). Diesel exhaust particles (DEPs) with a mean size of about $0.2 \mu \mathrm{m}$ are a major component of ambient $\mathrm{PM}_{2.5}$ (Sawyer \& Johnson, 1995). To further study the causal 
relationship between PM and the acute CHF, we used echocardiography to investigate whether DEPs cause left ventricular (LV) dysfunction in isoproterenol (ISO)-induced myocardial injury and healthy rats.

CHF has emerged as one of the most pressing public health issues (Jessup et al., 2003). It is a progressive disorder that often begins with LV dysfunction (Pfeffer et al., 1990; Mann, 1999). Echocardiography has long been used for assessing cardiac function and structure in humans with heart failure. Recently, transthoracic echocardiography with high-frequency imaging has been used to ascertain cardiac dimensions and function in small animals (Tanaka et al., 1996; Kido et al., 2005; Wikstrom et al., 2005). In this study, we applied Mmode echocardiography to assess LV function, including fractional shortening (FS) and left ventricular end-diastolic diameter (LVDd).

Since PM-related mortality and morbidity have usually occurred in susceptible populations, animal models of cardiopulmonary diseases have been used to investigate this causal relationship and its underlying mechanisms (Kodavanti et al., 1998). ISO is a beta agonist that causes myocardial oxygen demand to exceed supply and induces myocardial necrosis at high doses. Thus, we used this as a model of CHF (Grimm et al., 1998). This model is characterized by a technical simplicity and an excellent reproducibility, as well as an acceptable mortality rate (Pfeffer et al., 1990).

In this study, we used echocardiography to quantify the LV function in isoproterenol-induced myocardial injury and healthy rats. Our hypothesis is that acute DEPs exposure induces LV dysfunction, and this LV function is further compromised in rats with preexisting ISO-induced myocardial injury.

\section{METHODS}

\section{Animal Preparation and Exposure}

Male Sprague-Dawley rats weighing 150-200 g were obtained from Charles River Co., Ltd. They were housed in plastic cages on Aspen chip bedding and provided with Lab Diet 5001 and water ad libitum. Animals were maintained on a 12-h light/dark cycle at $22 \pm 1{ }^{\circ} \mathrm{C}$ and $55 \pm 10 \%$ relative humidity. Rats received subcutaneous injection of ISO (Sigma Chemical Co., $150 \mathrm{mg} / \mathrm{kg}$ body weight, dissolved in normal saline) to induce myocardial injury (Grimm et al., 1998), or normal saline as controls. Seven days later, both groups were further assigned to receive either $250 \mu \mathrm{g}$ DEPs in $0.5 \mathrm{ml}$ normal saline (SRM2975, National Institute of Standards Technology, USA) or a similar amount of normal saline by intratracheal instillation (IT). The DEPs doses, which were expected to cause lung inflammation and possible subsequent systemic inflammation and endothelial dysfunction, were based on our experience. Overall, 12 rats ( $n=6$ for both ISO and healthy rats) were anesthetized with pentobarbital $(50 \mathrm{mg} / \mathrm{kg}$ body weight $)$ and then instilled with DEPs intratracheally. The other 10 rats $(n=6$ for ISO; $n=4$ for healthy rats) were instilled with normal saline as controls. All protocols used in this experiment were approved by the National Taiwan University's animal care and use committee.

\section{Echocardiographic Evaluation of LV Function}

Pentobarbital was administered intraperitoneally at $50 \mathrm{mg} / \mathrm{kg}$ of body weight before the echocardiographic evaluation. Echocardiography conducted by one well-trained physician was performed 1 day before and after DEPs or normal saline exposure to assess the cardiac structure and function in experimental animals. LV function was assessed in vivo by transthoracic echocardiographic examinations using a 10$\mathrm{MHz}$ electronic probe (System V, GE, Chicago). Longitudinal imaging was performed at approximately $45^{\circ}$ through the left parasternal rib space with a maximum imaging depth of $30 \mathrm{~mm}$. End-systole or end-diastole was defined as the phase in which the smallest or largest area of LV was obtained. LV end-systolic diameter (LVDs) and LV end-diastolic diameter (LVDd) were measured at the level of the papillary muscle by the leadingedge method from at least three consecutive cardiac cycles on the M-mode tracing as suggested by the American Society for Echocardiography (Gottdiener et al., 2004). Linear measurements of fractional shortening (FS) were calculated as follows: FS $(\%)=100 \times[$ LVDd - LVDs $] /$ LVDd.

\section{Statistical Analysis}

Results were expressed as means \pm SE. Paired and unpaired $t$-tests were used to compare the differences within groups and between groups, respectively. Those $p$ values $<.05$ were considered significant. The entire statistical analysis was performed using SAS 8.2.

\section{RESULTS}

\section{Changes of FS and LVDd After ISO Injection}

FS was significantly decreased in healthy rats treated with ISO as compared to those with normal saline $(55.3 \%$ vs. $43.3 \%$, $p<.0001, t$-test). LVDd was higher in healthy rats treated with ISO as compared to those with normal saline; however, this relationship was not statistically significant.

\section{Effect of DEPs on FS and LVDd in ISO and Healthy Rats}

FS was further compared before and after DEPs or normal saline exposure in healthy and ISO rats (Figure 1). There was no difference after normal saline treatment in healthy and ISO groups. However, FS was significantly lower after DEPs exposure in both groups ( $p<.05$, paired $t$-test). Additionally, LVDd was higher in healthy rats, but not in ISO rats (Figure 2).

\section{Comparison of FS in Experimental Groups With a Different Combination of ISO and DEPs}

When using SD rats treated with normal saline as a reference group, SD rats treated with DEPs $(p<.05, t$-test $)$, and ISO rats treated with normal saline $(p<.0001, t$-test $)$ had 

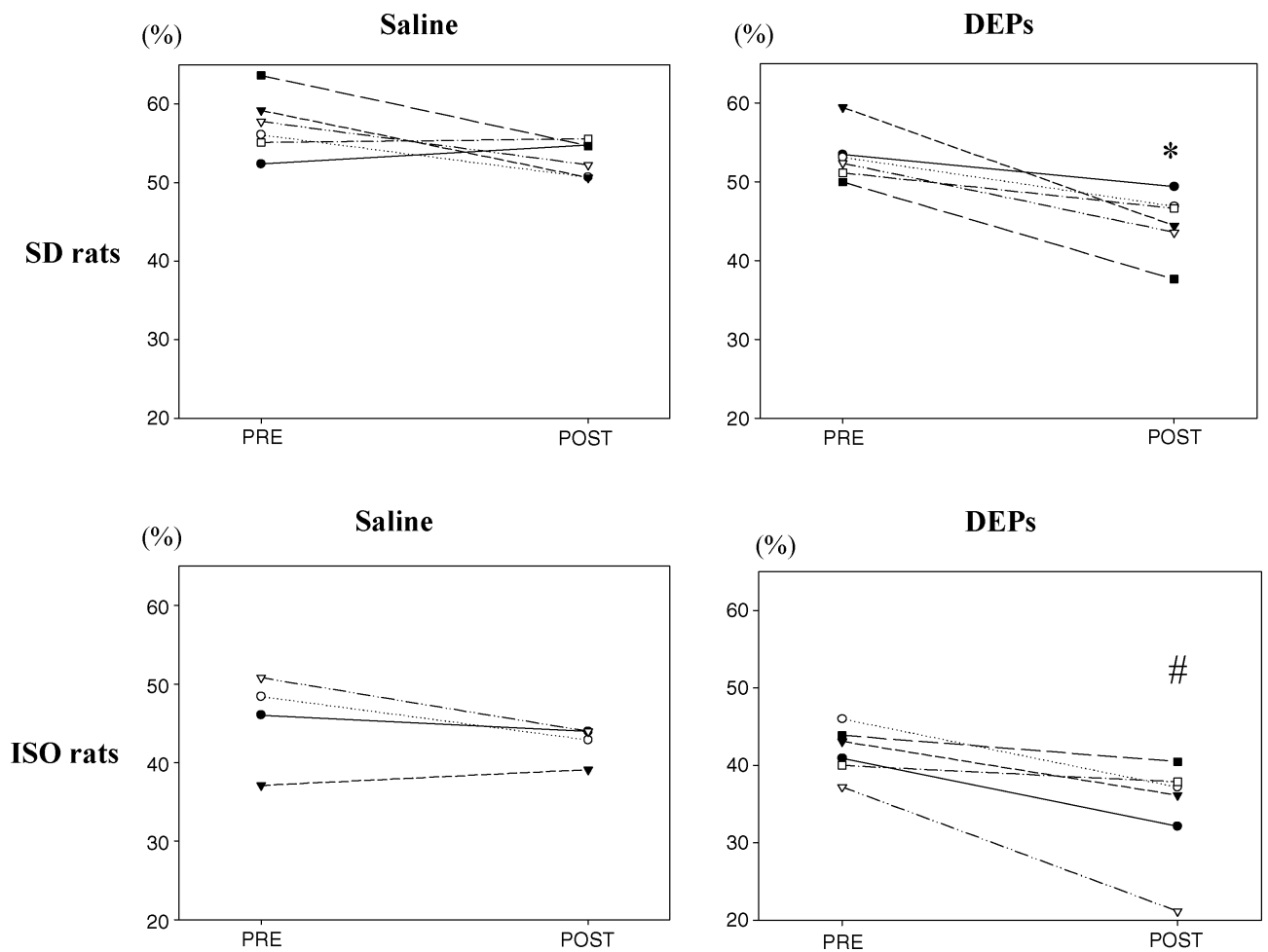

FIG. 1. Fractional shortenings before and after treatment; * indicates significant difference at $p=.01$ and \# at $p=.03$ vs pretreatment.
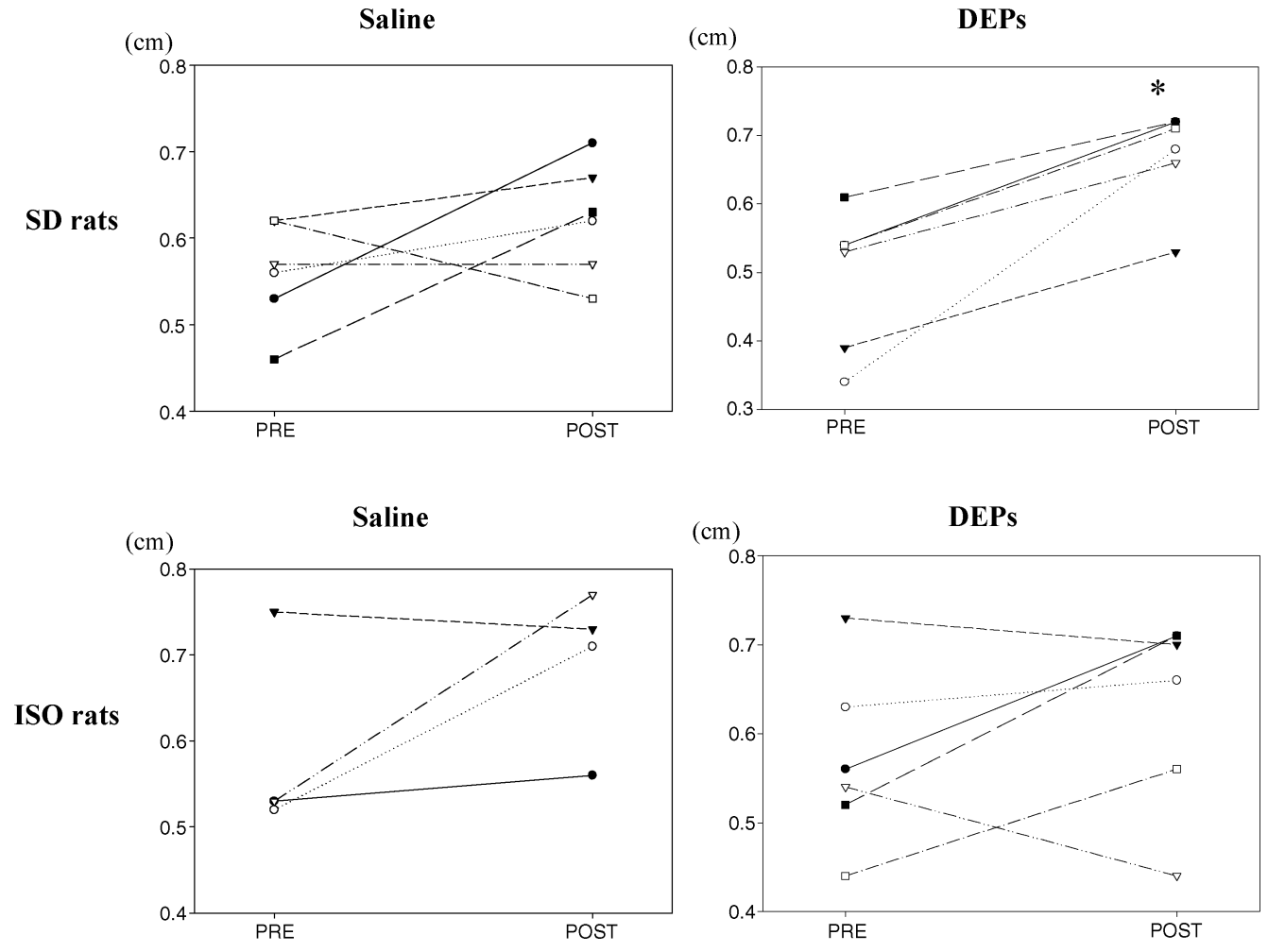

FIG. 2. Left ventricular end diastolic diameter before and after treatment; $*$ indicates significant difference at $p=.003$ vs pretreatment. 
TABLE 1

Fractional shortening (FS) and left ventricular end-diastolic diameter (LVDd) assessed by echocardiography before and after IT DEPs in isoproterenol (ISO)-induced myocardial injury rats and healthy SD rats

\begin{tabular}{|c|c|c|c|c|c|c|c|c|}
\hline & \multicolumn{4}{|c|}{ SD rats } & \multicolumn{4}{|c|}{ ISO rats } \\
\hline & $\begin{array}{c}\text { Pre } \\
(N=6)\end{array}$ & $\begin{array}{c}\text { Post } \\
(N=6)\end{array}$ & $\begin{array}{c}\text { Pre } \\
(N=6)\end{array}$ & $\begin{array}{c}\text { Post } \\
(N=6)\end{array}$ & $\begin{array}{c}\text { Pre } \\
(N=4)\end{array}$ & $\begin{array}{c}\text { Post } \\
(N=4)\end{array}$ & $\begin{array}{c}\text { Pre } \\
(N=6)\end{array}$ & $\begin{array}{c}\text { Post } \\
(N=6)\end{array}$ \\
\hline $\mathrm{FS}(\%)$ & $57.36 \pm 3.86$ & $53.09 \pm 2.19$ & $53.27 \pm 3.30$ & $44.77 \pm 4.04^{a, c}$ & $45.57 \pm 2.99$ & $42.46 \pm 2.31$ & $41.85 \pm 3.12$ & $34.16 \pm 6.93^{b, d, e, f}$ \\
\hline $\operatorname{LVDd}(\mathrm{cm})$ & $0.56 \pm 0.06$ & $0.62 \pm 0.07$ & $0.49 \pm 0.10$ & $0.67 \pm 0.07^{a}$ & $0.58 \pm 0.05$ & $0.69 \pm 0.09$ & $0.57 \pm 0.10$ & $0.63 \pm 0.11$ \\
\hline
\end{tabular}

${ }^{a}$ Significant at $p<.05$ compared to pre-IT DEPs in SD rats.

${ }^{b}$ Significant at $p<.05$ compared to pre-IT DEPs in ISO rats.

${ }^{c}$ Significant at $p<.05$ compared to post-IT saline in SD rats.

${ }^{d}$ Significant at $p<.05$ compared to post-IT saline in ISO rats.

${ }^{e}$ Significant at $p=.01$ compared to post-IT DEPs in SD rats.

${ }^{f}$ Significant at $p<.0001$ compared to post-IT saline in SD rats.

lower FS, while ISO rats treated with DEPs had the lowest FS $(p<.0001, t$-test) (Table 1). It appeared that there was an additive effect between ISO and DEPs exposure on FS.

\section{DISCUSSION}

The principal finding of this study is that DEPs impaired left ventricular function, and that LV function was further decreased in ISO-induced myocardial injury rats as revealed through echocardiography.

Echocardiography has long been used for assessing cardiac function and structure in humans with CHF. Although M-modederived linear measurements have the disadvantage of only determining ventricular function along a single interrogation line, the resolution of M-mode echocardiography is superior to that of two-dimensional echocardiography for precise identification of timing. The ability to visualize the entire left ventricle and to ensure a true minor-axis dimension mitigates these potential advantages (Harvey et al., 2004). Our study has demonstrated that echocardiography may be useful in assessing PM related cardiac LV function in rats.

To the best of our knowledge, this is the first experimental study to demonstrate that PM may impair LV function. Although we found that DEPs might cause decreased FS, the exact mechanisms are not clear. We speculate that PM-related decompensation of CHF may be mediated through the increase of sympathetic activity and alterations of vascular tone (Brook et al., 2002; Pope et al., 2004; Chang et al., 2005; Mills et al., 2005).

Since this study was only conducted with echocardiography, biomarkers or histological examination of CHF are needed to confirm the findings in the future study. Furthermore, hemodynamic monitoring during DEPs exposure is also suggested to understand the underlying mechanisms of PM related CHF. In addition, this study only determined acute effects of DEPs exposure. The chronic effects of DEPs on CHF also need to be investigated. The other limitation of this study is that we used intratracheal instillation to expose animals in this study. A pre- vious study has shown that there are similarity and difference in pulmonary toxicity between inhalation and intratracheal instillation (Costa et al., 2006). Thus, future study by inhalation is also suggested.

The PM effects were similar in ISO and healthy rats in this study. Thus, there was no potentiation of PM in ISO rats. It is possible that at current $\mathrm{LV}$ function levels there is adequate compensation for PM effects in ISO rats. Although the effects of PM were not greater in ISO rats as compared to healthy rats, there were additive effects between PM and ISO treatment. Thus, the LV dysfunction in ISO rats was most prominent among all groups. This implies that individuals with compensated $\mathrm{CHF}$ may be pushed over into decompensated CHF, requiring hospitalization, with acute exposure to fine PM. Our results support previous epidemiological findings on the association between fine PM and hospital admissions of CHF.

\section{REFERENCES}

Brook, R. D., Brook, J. R., Urch, B., Vincent, R., Rajagopalan, S., and Silverman, F. 2002. Inhalation of fine particulate air pollution and ozone causes acute arterial vasoconstriction in healthy adults. Circulation 105:1534-1536.

Brook, R.D., Franklin, B., Cascio, W., Hong, Y., Howard, G., Lipsett, M., Luepker, R., Mittleman, M., Samet, J., Smith, S. C., Jr., and Tager, I. 2004. Air pollution and cardiovascular disease: A statement for healthcare professionals from the Expert Panel on Population and Prevention Science of the American Heart Association. Circulation 109:2655-2671.

Chang, C. C., Hwang, J. S., Chan, C. C., Wang, P. Y., Hu, T. H., and Cheng, T. J. 2005. Effects of concentrated ambient particles on heart rate variability in spontaneously hypertensive rats. J. Occup. Health 47:471-480.

Costa, D. L., Lehmann, J. R., Winsett, D., Richards, J., Ledbetter, A. D., and Dreher, K. L. 2006. Comparative pulmonary toxicological assessment of oil combustion particles following inhalation or instillation exposure. Toxicol. Sci. 91:237-246.

Dominici, F., Peng, R. D., Bell, M.L., Pham, L., McDermott, A., Zeger, S. L., and Samet, J. M. 2006. Fine particulate air pollution and 
hospital admission for cardiovascular and respiratory diseases. $J$. Am. Med. Assoc. 295:1127-1134.

Gottdiener, J. S., Bednarz, J., Devereux, R., Gardin, J., Klein, A., Manning, W. J., Morehead, A., Kitzman, D., Oh, J., Quinones, M., Schiller, N. B., Stein, J. H., and Weissman, N. J. 2004. American Society of Echocardiography recommendations for use of echocardiography in clinical trials. J. Am. Soc. Echocardiogr. 17:1086-1119.

Grimm, D., Elsner, D., Schunkert, H., Pfeifer, M., Griese, D., Bruckschlegel, G., Muders, F., Riegger, G. A., and Kromer, E.P. 1998. Development of heart failure following isoproterenol administration in the rat: Role of the renin-angiotensin system. Cardiovasc. Res. 37:91-100.

Harvey, F., William, F. A., and Thomas, R. 2004. Feigenbaum's echocardiograph, p. 138. Philadelphia: Lippincott Williams \& Wilkins.

Jessup, M., and Brozena, S. 2003. Heart failure. N. Engl. J. Med. 348:2007-2018.

Kido, M., Du, L., Sullivan, C. C., Li, X., Deutsch, R., Jamieson, S. W., and Thistlethwaite, P. A. 2005. Hypoxia-inducible factor 1-alpha reduces infarction and attenuates progression of cardiac dysfunction after myocardial infarction in the mouse. J. Am. Coll. Cardiol. 46:2116-2124.

Kodavanti, U. P., Costa, D. L., and Bromberg, P. A. 1998. Rodent models of cardiovascular disease: Their potential applicability in studies of air pollutant susceptibility. Environ. Health Perspect. 106:111-130.

Koken, P. J., Piver, W. T., Ye, F., Elixhauser, A., Olsen, L. M., and Portier, C. J. 2003. Temperature, air pollution, and hospitalization for cardiovascular diseases among elderly people in Denver. Environ. Health Perspect. 111:1312-1317.

Mann, D. L. 1999. Mechanisms and models in heart failure: A combinatorial approach. Circulation 100:999-1008.

Metzger, K. B., Tolbert, P. E., Klein, M., Peel, J. L., Flanders, W. D., Todd, K., Mulholland, J. A., Ryan, P. B., and Frumkin, H. 2004. Ambient air pollution and cardiovascular emergency department visits. Epidemiology 15:46-56.

Mills, N. L., Tornqvist, H., Robinson, S.D., Gonzalez, M., Darnley, K., MacNee, W., Boon, N. A., Donaldson, K., Blomberg, A., Sandstrom, T., and Newby, D. E. 2005. Diesel exhaust inhalation causes vascular dysfunction and impaired endogenous fibrinolysis. Circulation 112:3930-3935.
Morris, R. D., and Naumova, E. N. 1998. Carbon monoxide and hospital admissions for congestive heart failure: evidence of an increased effect at low temperatures. Environ. Health Perspect. 106:649653.

Peters, A. 2005. Particulate matter and heart disease: Evidence from epidemiological studies. Toxicol. Appl. Pharmacol. 207:47-482.

Pfeffer, M. A., and Braunwald, E. 1990. Ventricular remodeling after myocardial infarction. Experimental observations and clinical implications. Circulation 81:1161-1172.

Pope, C. A. 3rd, Hansen, M. L., Long, R. W., Nielsen, K. R., Eatough, N. L., Wilson, W. E., and Eatough, D. J. 2004. Ambient particulate air pollution, heart rate variability, and blood markers of inflammation in a panel of elderly subjects. Environ. Health Perspect. 112:339-345.

Sawyer, R. F., and Johnson J. H. 1995. Diesel emissions and control technology. In Diesel exhaust: A critical analysis of emissions, exposure and health effects, pp. 67-81. Cambridge, MA: Health Effects Institute.

Schwartz, J., and Morris, R. 1995. Air pollution and hospital admissions for cardiovascular disease in Detroit, Michigan. Am. J. Epidemiol. 142:23-35.

Symons, J.M., Wang, L., Guallar, E., Howell, E., Dominici, F., Schwab, M., Ange, B. A., Samet, J., Ondov, J., Harrison, D., and Geyh A. 2006. A case-crossover study of fine particulate matter air pollution and onset of congestive heart failure symptom exacerbation leading to hospitalization. Am. J. Epidemiol. 164:421-433.

Tanaka, N., Dalton, N., Mao, L., Rockman, H. A., Peterson, K. L., Gottshall, K. R., Hunter, J. J., Chien, K. R., and Ross, J., Jr. 1996. Transthoracic echocardiography in models of cardiac disease in the mouse. Circulation 94:1109-1117.

Wellenius, G. A., Bateson, T. F., Mittleman, M. A., and Schwartz, J. 2005. Particulate air pollution and the rate of hospitalization for congestive heart failure among medicare beneficiaries in Pittsburgh, Pennsylvania. Am. J. Epidemiol. 161:1030-1036.

Wellenius, G. A., Schwartz, J., and Mittleman, M. A. 2006. Particulate air pollution and hospital admissions for congestive heart failure in seven United States cities. Am. J. Cardiol. 97:404-408.

Wikstrom, J., Gronros, J., Bergstrom, G., and Gan, L. M. 2005. Functional and morphologic imaging of coronary atherosclerosis in living mice using high-resolution color Doppler echocardiography and ultrasound biomicroscopy. J. Am. Coll. Cardiol. 46:720-726. 
Copyright of Inhalation Toxicology is the property of Taylor \& Francis Ltd and its content may not be copied or emailed to multiple sites or posted to a listserv without the copyright holder's express written permission. However, users may print, download, or email articles for individual use. 